\title{
Binding features by relaying modulator group of neurons
}

\author{
Toomas Kirt, Talis Bachmann \\ From Twentieth Annual Computational Neuroscience Meeting: CNS*2011 \\ Stockholm, Sweden. 23-28 July 2011
}

Simultaneous oscillation of neuron groups could provide solution to the binding problem [1] and simultaneously firing neurons could bind together different features of the same object. The corticothalamic system plays a key role in synchronizing the activity of thalamic and cortical neurons [2] and synchronized oscillations have important function in brain activities [3]. It is shown that a relaying neuron group could cause zero time lag synchronization among distant neuron groups [4]. As there are many fields representing different features then they compete with each other to become dominant; thus, Jörg Lücke [5] proposed that there might be multiple bifurcation points where the network tends to move towards a certain stable state. Such selection is achieved by the decision making processes [6], where a common group of inhibitory neurons inhibits activity of the to-be-loosing excitatory neuron group. Based on this we have built up an experimental setup where we used two pairs of exclusive features from two dimensions and tried to bind together one from each dimension as a unified object and cause activity of the higher level group of neurons.

In our experiments the Brian simulator [7] featuring a leaky integrate-and-fire neuron model is used and three main groups of excitatory neurons are formed. The first group is the modulator group (see Figure 1A Tc) which synchronizes activity of the next feature group (Py1) and the latter sends synaptic effects to the higher

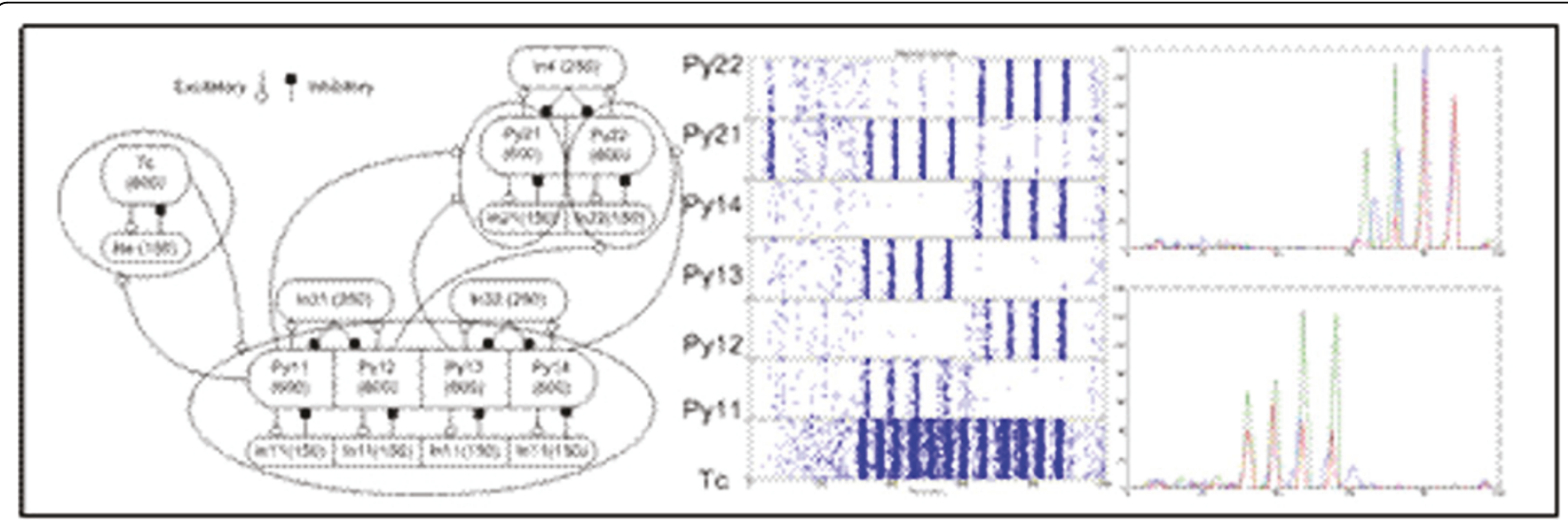

Figure 1 A) Network structure. B) Raster plot of spikes of excitatory neurons. C) Averaged firing histogram of subpopulations. Upper figure Py12 and Py 14 subpopulations and their common firing (blue, green and red respectively) and lower figure Py 11 and Py13 (the same color scheme).

\footnotetext{
* Correspondence: Toomas.Kirt@mail.ee

Laboratory of Cognitive Psychology, University of Tartu, Teatri väljak 3,

Tallinn 10143, Estonia

Full list of author information is available at the end of the article
}

C Biomed Central

(c) 2011 Kirt and Bachmann; licensee BioMed Central Ltd. This is an open access article distributed under the terms of the Creative Commons Attribution License (http://creativecommons.org/licenses/by/2.0), which permits unrestricted use, distribution, and reproduction in any medium, provided the original work is properly cited. 
consolidating group (Py2). All the excitatory neurons are connected to a local inhibitory group (Re, In1, In2) and the feature neurons and consolidating neurons get pairwise additional inhibitory input (In3, In4). All the neurons receive Poissonian background input noise that causes averaged network activity in the frequency of 1 $\mathrm{Hz}$. The neurons from modulator and feature groups get additional Poissonian input in a certain time frame and, during the simulations, activity switches from one pair of features to the other. As a result we could see the well synchronized activity of feature neurons could cause oscillating activity of neurons in the higher group (Figure 1).

There might be some number of basic mechanisms that are responsible for processing and forwarding information in the brain, including a variety of synchronizing mechanisms. In this experiment we have shown how synchronized oscillations of neurons and decision making processes can be used for binding and forwarding information between the neuron groups.

\section{Acknowledgements}

Supported by grant no. MJD22 financed with the support of the European Social Fund and by the ESF grant \#8401.

Published: 18 July 2011

\section{References}

1. von Der Malsburg C: The correlation theory of brain function. In Models of Neural Networks II: Temporal Aspects of Coding and Information Processing in Biological Systems. Berlin/New York: Springer;Domany E, Leonard Hemmen J, Schulten K 1994:95-119.

2. Jones EG: Synchrony in the interconnected circuitry of the thalamus and cerebral cortex. Annals of the New York Academy of Sciences 2009, 1157:10-23.

3. Buzsáki G: Rhythms of the Brain. New York: Oxford University Press; 2006.

4. Vicente R, Gollo LL, Mirasso CR, Fischer I, Pipa G: Dynamical relaying can yield zero time lag neuronal synchrony despite long conduction delays. Proceedings of the National Academy of Sciences of the United States of America 2008, 105:17157-17162.

5. Lücke J: Receptive field self-organization in a model of the fine structure in v1 cortical columns. Neural computation 2009, 21:2805-2845.

6. Wong KF, Wang XJ: A recurrent network mechanism of time integration in perceptual decisions. The Journal of neuroscience : the official journal of the Society for Neuroscience 2006, 26:1314-1328.

7. Goodman D, Brette R: Brian: a simulator for spiking neural networks in python. Frontiers in Neuroinformatics 2008, 2:1-10.

doi:10.1186/1471-2202-12-S1-P7

Cite this article as: Kirt and Bachmann: Binding features by relaying modulator group of neurons. BMC Neuroscience 2011 12(Suppl 1):P7.

\section{Submit your next manuscript to BioMed Central and take full advantage of:}

- Convenient online submission

- Thorough peer review

- No space constraints or color figure charges

- Immediate publication on acceptance

- Inclusion in PubMed, CAS, Scopus and Google Scholar

- Research which is freely available for redistribution

Submit your manuscript at www.biomedcentral.com/submit
C Biomed Central 